\title{
Retinoic Acid Response Element in the Human Alcohol Dehydrogenase Gene $A D H 3$ : Implications for Regulation of Retinoic Acid Synthesis
}

\author{
GREGG DUESTER,* MARY LOU SHEAN, M. SCOTT McBRIDE, † AND MARK J. STEWART $\ddagger$ \\ Department of Biochemistry, Colorado State University, Fort Collins, Colorado 80523
}

Received 9 October 1990/Accepted 18 December 1990

\begin{abstract}
Retinoic acid regulation of one member of the human class I alcohol dehydrogenase (ADH) gene family was demonstrated, suggesting that the retinol dehydrogenase function of ADH may play a regulatory role in the biosynthetic pathway for retinoic acid. Promoter activity of human $A D H 3$, but not $A D H 1$ or $A D H 2$, was shown to be activated by retinoic acid in transient transfection assays of Hep3B human hepatoma cells. Deletion mapping experiments identified a region in the $A D H 3$ promoter located between -328 and -272 bp which confers retinoic acid activation. This region was also demonstrated to confer retinoic acid responsiveness on the $A D H 1$ and $A D H 2$ genes in heterologous promoter fusions. Within a 34-bp stretch, the $A D H 3$ retinoic acid response element (RARE) contains two TGACC motifs and one TGAAC motif, both of which exist in RAREs controlling other genes. A block mutation of the TGACC sequence located at $\mathbf{2 8 9}$ to $\mathbf{- 2 8 5}$ bp eliminated the retinoic acid response. As assayed by gel shift DNA binding studies, the RARE region (-328 to -272 bp) of $A D H 3$ bound the human retinoic acid receptor $\beta(R A R \beta)$ and was competed for by DNA containing a RARE present in the gene encoding RARB. Since ADH catalyzes the conversion of retinol to retinal, which can be further converted to retinoic acid by aldehyde dehydrogenase, these results suggest that retinoic acid activation of $A D H 3$ constitutes a positive feedback loop regulating retinoic acid synthesis.
\end{abstract}

Retinoic acid has profound effects on vertebrate limb and nervous system morphogenesis as well as epithelial cell differentiation $(12,31,40)$. The effects of retinoic acid are transduced by binding to a nuclear retinoic acid receptor (RAR) which, in the presence of ligand, is transformed into a transcription factor $(6,14,28)$. Recently, a RAR gene family $(\operatorname{RAR} \alpha,-\beta$, and $-\gamma)$ has been discovered $(3,49)$, and differential expression of these receptors is undoubtedly important for correct transduction of the retinoic acid signal in various target tissues (9). There also exists another nuclear retinoic acid receptor, designated $R X R \alpha$, which is not part of the RAR family and which is expressed highly in liver (23). Genes that respond to retinoic acid have been identified, and characterization of the retinoic acid response element (RARE) has been accomplished for genes encoding growth hormone (42), laminin B1 (45), RAR $\beta$ (7), and osteocalcin (33).

One important aspect of retinoic acid-induced differentiation that is not understood is the mechanism regulating synthesis of retinoic acid from retinol (vitamin A). In mammals, retinol is reversibly oxidized to retinal by a cytosolic retinol dehydrogenase that is identical to class $\mathrm{I}$ alcohol dehydrogenase (ADH; EC 1.1.1.1), the enzyme which also functions as an ethanol dehydrogenase $(21,22,26,29,44$, 48). Human ADH consists of a group of isozymes divided into three classes (I, II, and III) based on structural and functional distinctions (43). Human class I ADH, which has been reported to oxidize ethanol and retinol $(26,43)$, consists of homodimers and heterodimers of the $\alpha, \beta$, and $\gamma$ polypep-

\footnotetext{
* Corresponding author.

$\uparrow$ Present address: McArdle Laboratory for Cancer Research, University of Wisconsin, Madison, WI 53706.

$\ddagger$ Present address: Department of Biological Chemistry, Howard Hughes Medical Institute, University of Michigan Medical Center, Ann Arbor, MI 48109-0650.
}

tide subunits encoded by three closely related genes $A D H I$, $A D H 2$, and $A D H 3$, respectively (34). The substrate specificities of the various class I ADH isozymes (i.e., $\alpha \alpha, \beta \beta, \gamma \gamma$, $\alpha \beta, \alpha \gamma$, and $\beta \gamma$ ) are very similar (43). Human ADH classes II and III are encoded by $A D H 4$ and $A D H 5$, respectively, and have been implicated in other metabolic reactions $(19,24)$. The reversible oxidation of retinol to retinal by class I ADH is followed by the irreversible oxidation of retinal to retinoic acid by an aldehyde dehydrogenase, and under in vivo conditions the retinol-retinal interconversion is the ratelimiting step in retinoic acid synthesis (22). Further support for class I ADH functioning as a physiologically relevant enzyme in controlling retinoic acid synthesis has come from studies on inhibition of retinol oxidation. Ethanol, a typical class I ADH substrate, acts as a competitive inhibitor of retinol oxidation in human liver extracts (26), and we have proposed a connection between fetal alcohol syndrome, retinoic acid homeostasis, and ethanol-retinol metabolism catalyzed by human ADH (10). In addition, ethanol and the class I ADH inhibitor 4-methylpyrazole have been shown to reduce retinol oxidation and retinoic acid synthesis in rodent liver $(21,29,44)$.

To gain more insight into the overall function of mammalian ADH and its role in the pathway for retinoic acid synthesis, we have undertaken studies to examine the transcriptional regulation of human $\mathrm{ADH}$ genes. The three human class I ADH genes display differential patterns of expression in many tissues throughout development, with highest expression occurring in adult liver $(2,34)$. The 5 '-flanking regions of human $A D H 1, A D H 2$, and $A D H 3$ have been characterized $(11,35)$. Activation of the $A D H 2$ promoter by the transcription factor CCAAT/enhancer-binding protein (36) as well as glucocorticoids (47) has been characterized in hepatoma cells. We now demonstrate that retinoic acid selectively activates the promoter for the $A D H 3$ gene in hepatoma cells and have characterized the RARE. This 


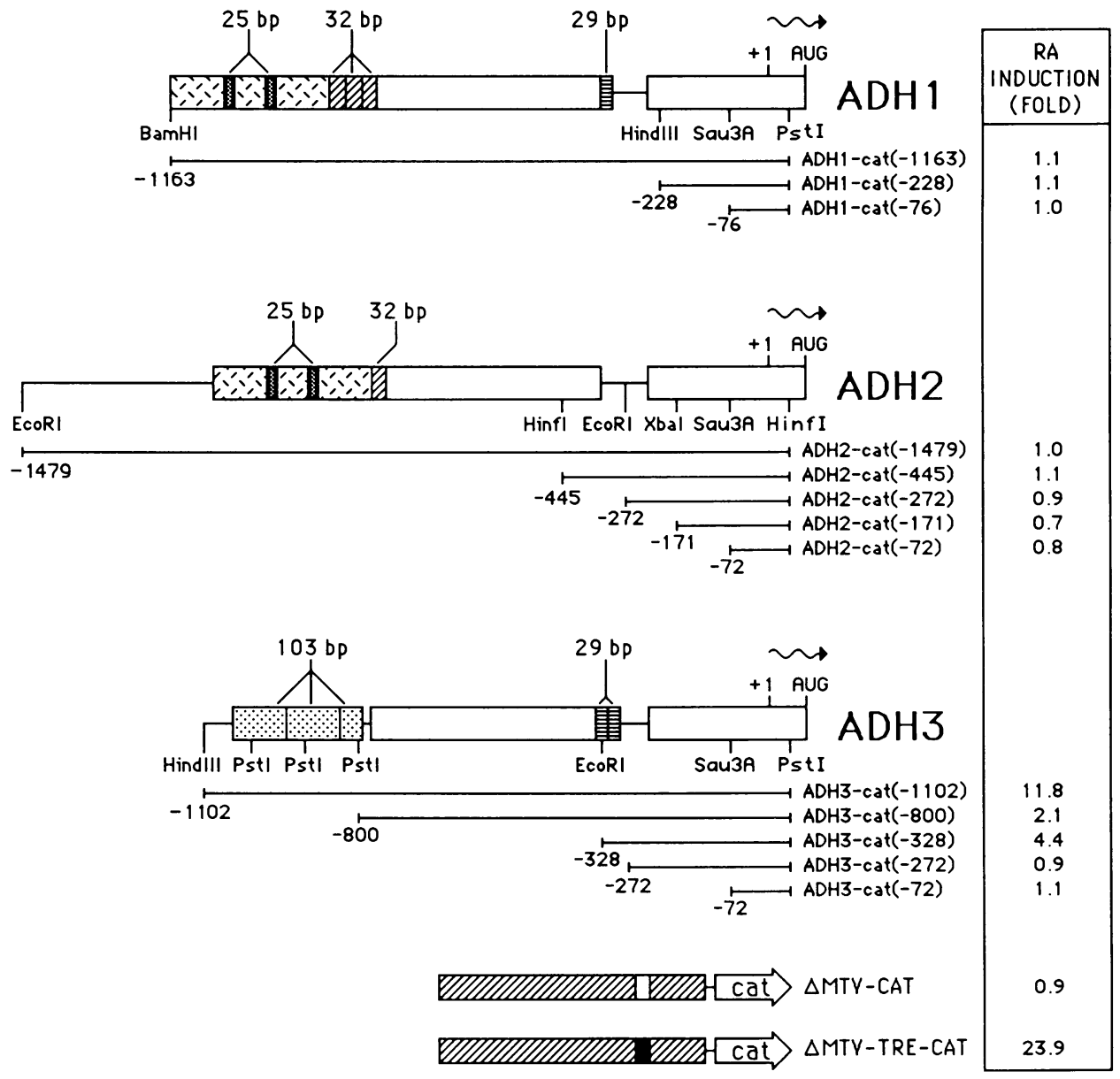

FIG. 1. Transcriptional responses of $A D H$ promoters to retinoic acid. The 5 '-flanking regions of the human $A D H 1, A D H 2$, and $A D H 3$ genes are shown, with various bar patterns indicating regions of homology or repeated sequences unique to only one gene or shared by two genes. The three genes have $>80 \%$ sequence identity in the regions indicated by open bars. The promoters were fused to cat, and the numbers on the $5^{\prime}$ ends indicate the upstream endpoints of the constructions. Diagrams of the control plasmids p $\Delta M T V-C A T$ and $\mathrm{p} \Delta \mathrm{MTV}-\mathrm{TRE}-\mathrm{CAT}$ are also shown. The open box in $\triangle \mathrm{MTV}$-CAT indicates a deletion of the glucocorticoid response element between -190 and $-88 \mathrm{bp}$, and the filled box in $\triangle$ MTV-TRE-CAT indicates the insertion of an oligonucleotide bearing a palindromic RARE based on the sequence of the growth hormone RARE (42). Plasmids were cotransfected into Hep3B cells with the RAR $\alpha$ expression plasmid pRShRR, and promoter activity was estimated by CAT assays. The fold induction for retinoic acid-treated versus nontreated cells is shown.

finding enables us to hypothesize that retinoic acid positively regulates its own synthesis by a classic feedback loop in which the product of the pathway (i.e., retinoic acid) regulates an enzymatic step in that pathway (i.e., ADH). This provides compelling evidence for human class I ADH playing a regulatory role in retinoic acid synthesis in vivo.

\section{MATERIALS AND METHODS}

Plasmid constructs and site-directed mutagenesis. Fusion of various lengths of the $5^{\prime}$-flanking sequences of the human $A D H 1, A D H 2$, and $A D H 3$ genes to the chloramphenicol acetyltransferase (CAT) gene (cat) has been described (35). The genomic sequences were derived from $\lambda \mathrm{ADH} 26$ for $A D H 1, \lambda \mathrm{ADH} 15$ for $A D H 2$, and $\lambda \mathrm{ADH} 8$ for $A D H 3$ (11). Genomic restriction sites (Fig. 1) were used to excise portions of the 5'-flanking DNA for subsequent fusion to cat, except for the construction $A D H 3-\operatorname{cat}(-272)$, in which a $B g$ III site was created at -272 bp by site-directed mutagenesis as described below. In all constructions, the AUG translation start codon of cat is the first such codon to appear downstream of the $A D H$ transcription start point.
Site-directed mutations were made in the $A D H 3$ 5'-flanking region by the method of Kunkel et al. (20). Site-directed mutations in plasmid $A D H 3-c a t(-328)$ which created $B g l I I$ sites at -290 and -272 bp formed plasmids $A D H 3-\operatorname{cat}(-328$ mutant 1) and $A D H 3$-cat $(-328$ mutant 2$)$, respectively (see Fig. 3). The presence of the appropriate mutation was confirmed by chemical DNA sequence analysis (25).

To generate a small DNA fragment containing the complete $A D H 3$ RARE, a 73-bp $B g$ III fragment was liberated from plasmid $A D H 3-c a t(-328$ mutant 2) which contains 16 bp of vector DNA and 57 bp of $A D H 3$ DNA between -328 to -272 bp (see Fig. 3d). This $A D H 3$ RARE was inserted upstream of the $A D H 1$ and $A D H 2$ promoters to form heterologous promoter constructions. One and two copies of the 73-bp BglII DNA fragment were inserted into a BamHI site located 3,090 bp upstream of the $A D H 1$ promoter in plasmid $A D H I$-cat (-228) to form plasmids $A D H I$-cat (-228 RAREx1) and $A D H I-c a t(-228$ RAREx2), respectively. One and two copies of the 73-bp $B g$ lII DNA fragment were also inserted into a $B g$ III site located 290 bp upstream of the $A D H 2$ promoter in plasmid $A D H 2-\operatorname{cat}(-272)$ to form plasmids 
$A D H 2-c a t(-272$ RAREx1) and $A D H 2$-cat(-272 RAREx2), respectively.

All plasmids used for transfection and DNA-binding studies were purified through two cycles of $\mathrm{CsCl}$ gradient centrifugation (32).

Cell culture and transfection analysis. Transfections and CAT assays were performed by a modification of previously described methods $(15,17)$. Hep3B cells $(18)$ were cultured in minimal essential medium supplemented with $10 \%$ fetal bovine serum. Cells were seeded in $100-\mathrm{mm}$ tissue culture dishes and the following day were changed to medium containing charcoal-stripped serum to remove endogenous retinoids $(1,16)$. Transfection by the calcium phosphate coprecipitate method was performed with a mixture of DNA including $15 \mu \mathrm{g}$ of a reporter cat plasmid, $20 \mu \mathrm{g}$ of carrier plasmid pUC18, and $5 \mu \mathrm{g}$ of internal control plasmid pCH110, which contains a simian virus 40 early promoterlac $Z$ fusion used to correct for plate-to-plate differences in transfection efficiency (17). For cotransfections with RAR $\alpha$, $5 \mu \mathrm{g}$ of the RAR expression plasmid pRShRR (14) replaced $5 \mu \mathrm{g}$ of the carrier pUC18 to keep the total amount of DNA constant at $40 \mu \mathrm{g}$. The DNA precipitate $(40 \mu \mathrm{g}$ total) was added to the cells for $16 \mathrm{~h}$, after which time the cells were washed and supplemented with medium containing charcoalstripped serum; where indicated, all-trans-retinoic acid was added at $10^{-7} \mathrm{M}$, a concentration that is 10 -fold higher than the $50 \%$ effective dose $\left(10^{-8} \mathrm{M}\right)$ for binding of retinoic acid to $\operatorname{RAR} \alpha(3,28)$. Cells were harvested $48 \mathrm{~h}$ after DNA addition, and cell extracts were prepared. Extracts were first assayed for $\beta$-galactosidase activity, and then appropriate amounts of extract normalized for $\beta$-galactosidase activity were used in the CAT assay. CAT assay reaction mixtures were incubated for $2 \mathrm{~h}$ at $37^{\circ} \mathrm{C}$, and the reaction products were analyzed by thin-layer chromatography. The amount of CAT activity (percent acetylation of chloramphenicol) was quantitated by cutting out substrate and product spots from the thin-layer chromatograms and counting the radioactivity by liquid scintillation. Reported values for retinoic acid induction were estimated as the mean of the ratio between retinoic acid-induced and noninduced CAT activity from three or more experiments.

DNA-binding gel mobility shift. Gel mobility shift assays were performed essentially as described by de The et al. (7). A nuclear extract derived from $\mathrm{HeLa}$ cells infected with a recombinant vaccinia virus carrying the RAR $\beta$ cDNA under the transcriptional control of the late promoter for the $11 \mathrm{~K}$ protein gene (7) was obtained from $\mathbf{H}$. Stunnenberg and $\mathbf{M}$. Vivanco-Ruiz (European Molecular Biology Laboratory, Heidelberg, Federal Republic of Germany). A control HeLa nuclear extract from uninfected cells was prepared by the method of Dignam et al. (8). An amount of nuclear extract corresponding to $4.5 \mu \mathrm{g}$ of total protein was mixed with 40 fmol (approximately 20,000 to $40,000 \mathrm{cpm}$ ) of a ${ }^{32} \mathrm{P}$-labeled DNA probe in a solution containing (final concentrations) 7.5\% glycerol, $0.05 \mathrm{mM}$ EDTA, $10 \mathrm{mM} \mathrm{N}$-2-hydroxyethylpiperazine- $N^{\prime}$-2-ethanesulfonic acid HEPES; (pH 7.9) 5.5 $\mathrm{mM} \mathrm{MgCl} 2,25 \mathrm{mM} \mathrm{KCl}, 3 \mathrm{mM}$ spermidine, and $0.1 \mathrm{mg}$ of poly $(\mathrm{dI}-\mathrm{dC})$ per $\mathrm{ml}$ in a final volume of $20 \mu \mathrm{l}$. The mixture was incubated at $0^{\circ} \mathrm{C}$ for $1 \mathrm{~h}$ and then loaded onto a $5 \%$ nondenaturing polyacrylamide gel (29:1 weight ratio of acrylamide to bisacrylamide) buffered with $50 \mathrm{mM}$ Trishydrochloride (pH 8.3), $50 \mathrm{mM}$ boric acid, and $1 \mathrm{mM}$ EDTA; before sample loading, the gel was prerun at $120 \mathrm{~V}$ for $1 \mathrm{~h}$ at room temperature $\left(25^{\circ} \mathrm{C}\right)$. Electrophoresis was continued at $120 \mathrm{~V}$ at room temperature for 2.5 to $3.5 \mathrm{~h}$, the gel was vacuum dried, and the dried gel was analyzed by autoradiography.
DNA probes for the gel mobility shift reactions included a 73-bp BglII fragment derived from plasmid $A D H 3-c a t(-328$ mutant 2) which contains $A D H 3$ DNA between -328 to $-272 \mathrm{bp}$ and a 55-bp $B g$ III fragment derived from $A D H 3-$ cat (-328 mutant 1$)$ which contains $A D H 3$ DNA between -328 to -290 bp (see Fig. 3c and d). A double-stranded oligonucleotide containing the RAR $\beta$ RARE from -59 to -33 bp served as a positive control DNA probe (7). As competitor DNA molecules, unlabeled RAR $\beta$ RARE oligonucleotide DNA or a nonspecific competitor oligonucleotide comprising the binding site for the transcription factor HNF-3 (5) was added (4 pmol) to some gel mobility shift reactions to provide a 100 -fold molar excess over labeled probe DNA.

\section{RESULTS}

Differential retinoic acid responses of the human class I ADH promoters. Nucleotide sequences of the human $A D H 1$, $A D H 2$, and $A D H 35^{\prime}$-flanking regions are known, and sequence homologies among them have been previously reported (35). Blocks of sequence homology are shown schematically in Fig. 1. The three genes have extensive sequence identity from the first exon upstream to approximately -250 $\mathrm{bp}$, at which point all three genes diverge such that $A D H 3$ possesses a 29-bp direct repeat, with $A D H I$ having one of the 29-bp sequences and $A D H 2$ having a region of poor homology to the 29-bp sequence (Fig. 1). Just upstream of the 29-bp region, all three genes regain extensive sequence identity until approximately $-800 \mathrm{bp}$, at which point the $A D H 3$ gene diverges and contains a 103-bp repeat, whereas the $A D H I$ and $A D H 2$ genes continue to be homologous as far upstream as the $A D H I$ gene sequence has been determined (Fig. 1).

To determine whether retinoic acid regulates any of the three class I ADH promoters, we performed transient transfection of the differentiated human hepatoma cell line Hep3B (18) with various plasmid constructions in which a deletion series of $A D H I, A D H 2$, or $A D H 35^{\prime}$-flanking DNA fragments were fused to the marker gene cat. For $A D H$-cat fusions, the names include a number in parentheses which indicates the upstream endpoint of the $A D H 5^{\prime}$-flanking DNA (Fig. 1). In these studies we have cotransfected $A D H$-cat fusions with the RAR expression plasmid pRShRR (14). This plasmid contains the cDNA encoding human $\operatorname{RAR} \alpha$ fused to the Rous sarcoma virus promoter and has been introduced into Hep3B cells to increase production of the receptor in order to facilitate analysis of retinoic acid induction. Two control plasmids were used to monitor retinoic acid regulation. Plasmid $\mathrm{p} \Delta \mathrm{MTV}$-TRE-CAT contains the mouse mammary tumor virus promoter upstream of cat fused to a palindromic sequence that serves the dual role of a thyroid hormone response element (TRE) and a RARE; plasmid $p \Delta M T V-C A T$ is missing the TRE/RARE and does not respond to retinoic acid (42). Of all constructions tested, expression of cat was significantly induced only when plasmids pDMTV-TRE-CAT, $A D H 3$-cat(-1102), and $A D H 3-$ cat $(-328)$ were transfected in the presence of both retinoic acid and RAR $\alpha$ (Fig. 2; see Fig. 1 for summary of DNAs tested). None of the $A D H I$-cat or $A D H 2$-cat fusions responded to retinoic acid (Fig. 1), suggesting that these genes are not controlled by retinoic acid. The basal level CAT activities of all $A D H$-cat fusions were very similar and relatively low, falling in the range of 0.1 to $0.2 \%$ acetylation after normalization to the cotransfected $\beta$-galactosidase activities. Expression of endogenous $A D H 1, A D H 2$, or $A D H 3$ 

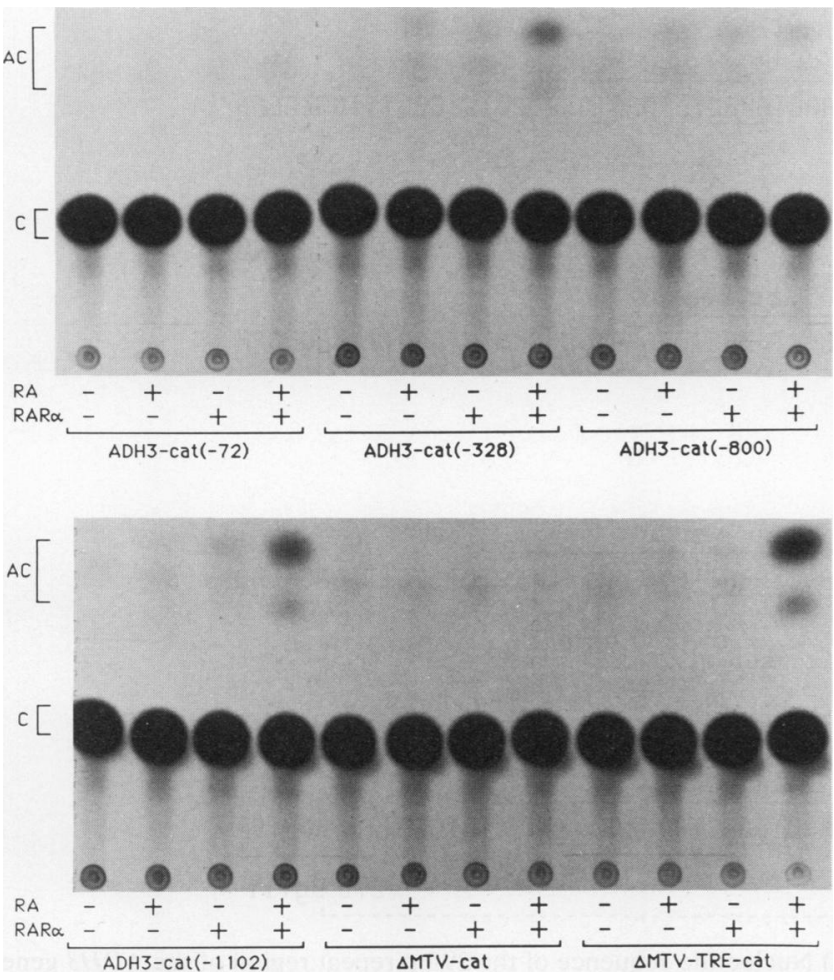

FIG. 2. CAT assays of a deletion series of ADH3-cat reporter genes. $A D H 3$-cat deletion series plasmids (see Fig. 1), as well as $\mathrm{p} \Delta \mathrm{MTV}$-CAT and $\mathrm{p} \Delta \mathrm{MTV}$-TRE-CAT (42), were transfected into Hep3B cells under the various conditions indicated. Thin-layer chromatograms showing the CAT activities are shown. RA, $10^{-7} \mathrm{M}$ retinoic acid; RAR $\alpha, 5 \mu \mathrm{g}$ of pRShRR RAR expression plasmid; C, chloramphenicol substrate; AC, acetylated chloramphenicol products.

mRNAs was undetectable in Northern (RNA) blots of total RNA from Hep3B cells grown in the absence of added retinoic acid (33a), thus correlating with the low CAT activity values of transfected ADH promoters. The lack of retinoic acid responsiveness of transfected $A D H 1$ and $A D H 2$ promoters is, however, not due to incompetence of these promoters in Hep3B cells, since both display induced promoter activity when fused to the $A D H 3$ RARE (see below).

$A D H 3$-cat (-1102) and $A D H 3-c a t(-328)$ exhibited retinoic acid inductions of 11.8 and 4.4 -fold, respectively (Fig. 1). The constructions $A D H 3-c a t(-272)$ and $A D H 3-c a t(-72)$ did not respond to retinoic acid (Fig. 1), suggesting that $A D H 3$ DNA between -328 to -272 bp may harbor a RARE. Interestingly, this region of $A D H 3$ overlaps the 29-bp sequence discussed above, which is directly repeated in $A D H 3$ but present only once in $A D H I$ and absent in $A D H 2$ (Fig. 1). The small retinoic acid induction (2.1-fold) exhibited by $A D H 3-c a t(-800)$ (Fig. 1 and 2) may be due to an unmasking of a negative element between -800 and -328 bp which is apparent only when sequences upstream of $-800 \mathrm{bp}$ are absent. A distal positively acting sequence between -1102 to $-800 \mathrm{bp}$ may relieve the negative effect of such a sequence located between -800 and $-328 \mathrm{bp}$. Since these studies narrowed the location of an $A D H 3$ RARE to between -328 to $-272 \mathrm{bp}$, we further characterized this region in detail as shown below, but additional RAREs upstream of -328 or downstream of +55 bp cannot be ruled out.

Site-directed mutagenesis of the $A D H 3$ RARE. In the $A D H 3$ promoter sequence (35), there is a 29-bp direct repeat between -340 and -283 bp ( 27 of 29 bp identical) that overlaps with the DNA sequence between -328 to $-272 \mathrm{bp}$ shown above to be part of the $A D H 3$ RARE (Fig. 3a). Each 29-bp repeat contains the sequence 5'-TGACC-3', previously noticed as an important element of the bifunctional TRE/RARE present in the growth hormone gene (42), as well as the RARE in the laminin B1 gene (45). The downstream 29-bp repeat also contains the related sequence TGAAC, which is present as a direct repeat in the RAREs of the human and mouse $\operatorname{RAR} \beta$ genes $(7,39)$. The function of the $A D H 3$ RARE was completely eliminated by creating a block mutation in plasmid $A D H 3-c a t(-328)$ which changed the downstream TGACC sequence (at -289 to $-285 \mathrm{bp}$ ) to GATCT (Fig. 3b and c). The fold retinoic acid induction of this mutant, $A D H 3$-cat $(-328$ mutant 1$)$, was 1.1 , compared with 4.4 for the wild-type $A D H 3-\operatorname{cat}(-328)$ (Fig. 4). This finding indicates that the downstream TGACC is an important part of the $A D H 3$ RARE and shows that the sequence requirements for this element appear to conform with that previously seen for RAREs in growth hormone, laminin B1, and RAR $\beta$ genes.

Fusion of heterologous promoters to an $A D H 3$ RARE. A $B g l$ II restriction fragment containing $A D H 3$ RARE DNA spanning -328 to -272 bp (Fig. $3 \mathrm{~d}$ ) was fused to heterologous promoters to determine whether it could impart retinoic acid responsiveness. The $A D H 3$ RARE was fused as one or two tandem copies upstream of the $A D H 1$ and $A D H 2$ 5 '-flanking regions in plasmids $A D H 1$-cat $(-228)$ and $A D H 2$ cat $(-272)$, respectively, both of which were shown above to be unresponsive to retinoic acid (Fig. 1). When one copy of the $A D H 3$ RARE was present, the construction $A D H 2$ cat $(-272$ RAREx1) responded well, with a retinoic acid induction of 3.8-fold, whereas the construction $A D H 1$ cat(-228 RAREx1) responded poorly, with a 1.4-fold induction (Fig. 4). When two copies were present, there were large inductions for both $A D H 2$-cat(-272 RAREx2) and ADHIcat(-228 RAREx2) of 13.5- and 19.8-fold, respectively (Fig. 4). This result provides further evidence that the $A D H 3$ sequence between -328 to -272 bp has the properties of a RARE, i.e., functioning as a retinoic acid-responsive enhancer element for a linked heterologous promoter.

Gel mobility shift using overexpressed RARß. To characterize the $A D H 3$ RARE further, we performed gel mobility shift DNA-binding studies using a nuclear extract derived from HeLa cells overexpressing human RAR $\beta$ in a vaccinia virus cDNA expression vector (7). A DNA fragment containing $A D H 3$ sequences spanning -328 to -272 bp was shifted in mobility by nuclear extract from vaccinia virusRAR $\beta$-infected HeLa cells such that a triplet of lowermobility bands was observed (Fig. 5a, lanes 1 and 2). However, a fragment containing sequences from -328 to -290 bp was not shifted in mobility (Fig. 5a, lanes 3 and 4). As a positive control, an oligonucleotide containing the previously characterized RARE in the RAR $\beta$ gene (7) was also shifted in mobility, giving a single band (Fig. 5a, lanes 5 and 6) with nearly the same mobility as the middle band of the triplet observed by using the longer $A D H 3$ probe (Fig. 5a, lane 2). Binding of the $A D H 3-328$ to -272 -bp fragment with vaccinia virus-RAR $\beta$ HeLa nuclear extract led to the appearance of a triplet of three bands exhibiting lower mobility than the free DNA, but when HeLa nuclear extract from cells uninfected with the vaccinia virus-RAR $\beta$ expression vector was used, only the upper and lower shifted bands were seen with the -328 to -272 -bp fragment as a probe (Fig. 5b; compare lanes 2 and 5). Thus, the middle band of 
a. ADH3 5'-flanking region

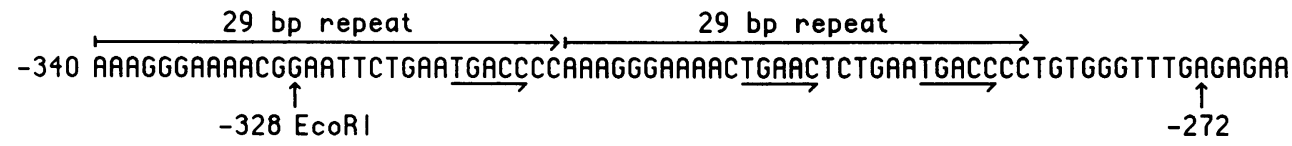

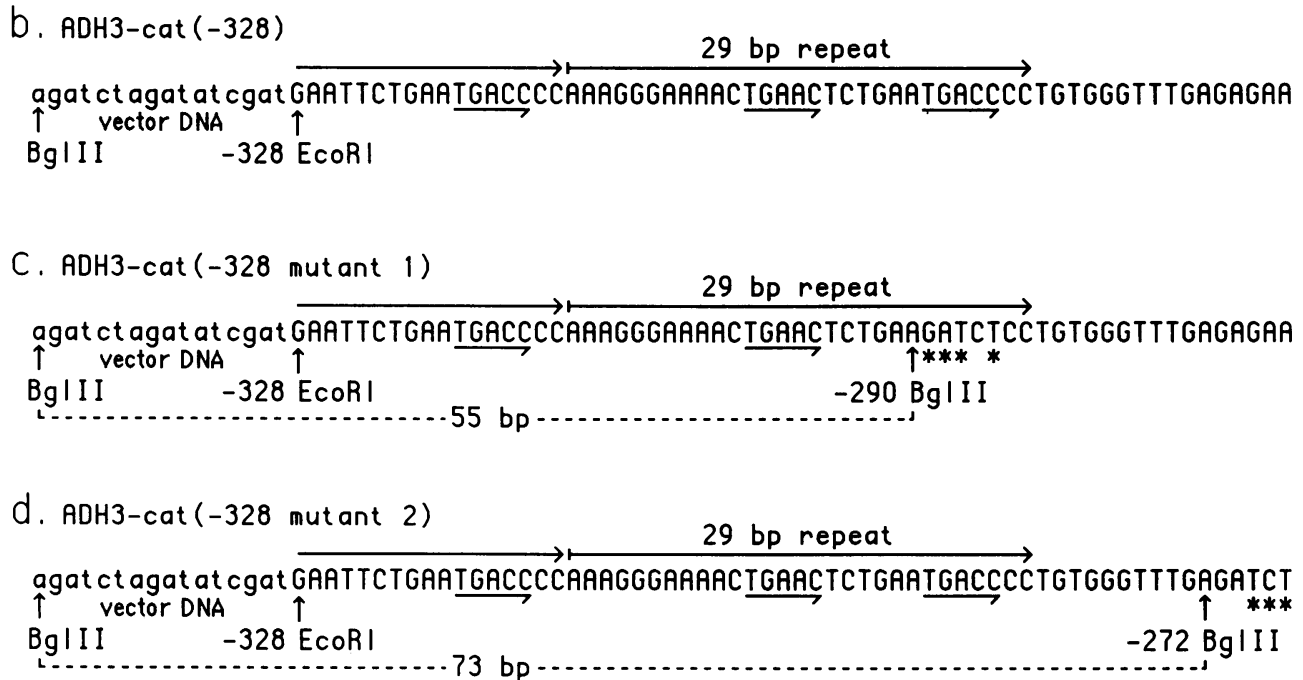

FIG. 3. Site-directed mutagenesis of the $A D H 3$ 29-bp repeat region. (a) Nucleotide sequence of the 29-bp repeat region of the $A D H 3$ gene (35). Two TGACC motifs and one TGAAC motif are indicated by small arrows. In the construction $A D H 3$-cat $(-328)(\mathrm{b})$, the upstream endpoint of $A D H 35^{\prime}$-flanking DNA is at an EcoRI site located at -328 bp and is shown fused to the cat vector DNA. Even though part of the first 29-bp repeat is missing in the fusion to the cat vector, all three RARE sequence motifs are present, as indicated by small arrows. The vector DNA sequence (shown in lowercase) contains a BglII site 16 bp upstream of the ADH3 DNA. ADH3-cat ( -328 mutant 1) (c) was formed by engineering a block substitution at -289 to -285 bp by site-directed mutagenesis which created a $B g l I I$ site at -290 bp; the base pairs mutated are indicated by asterisks. This eliminated the downstream TGACC motif and also enabled the isolation of a 55-bp BglII restriction fragment carrying two of the RARE motifs. ADH3-cat $(-328$ mutant 2) (d) was formed by engineering a block substitution at -269 to $267 \mathrm{bp}$ (indicated by asterisks) which created a BglII site at $-272 \mathrm{bp}$. This mutation was created to enable the isolation of a 73-bp BglII restriction fragment carrying all three RARE motifs.

the previously observed triplet is likely to represent a specific interaction between the $A D H 3$ probe and vaccinia virus-produced RAR $\beta$, whereas the other bands represent interactions with native HeLa factors that are presumably distinct from RAR $\beta$. When the RAR $\beta$ RARE oligonucleotide was added as a competitor, the middle band of the shifted triplet was completely eliminated and the upper and lower bands were greatly reduced in appearance (Fig. 5b, lanes 3 and 6). A nonspecific competitor, the binding site for the liver-specific transcription factor HNF-3 (5), did not compete any of the shifted bands (Fig. 5b, lanes 4 and 7).

These experiments indicate that the -328 to $-272-b p$ $A D H 3$ DNA fragment specifically binds RAR $\beta$ and that a critical component of the binding site lies between -290 to $-272 \mathrm{bp}$, since deletion of this segment eliminated binding of vaccinia virus-produced RAR $\beta$. These data correlate with the mutational analysis discussed above in which a block substitution of the TGACC motif between -289 to $-285 \mathrm{bp}$ eliminated retinoic acid responsiveness. These DNA-binding experiments suggest that the mechanism of $A D H 3$ promoter activation by retinoic acid described above is mediated by binding of the RAR to the $A D H 35^{\prime}$-flanking region.

\section{DISCUSSION}

In these studies, a RARE that regulates transcription of the human $A D H 3$ gene has been identified and character-

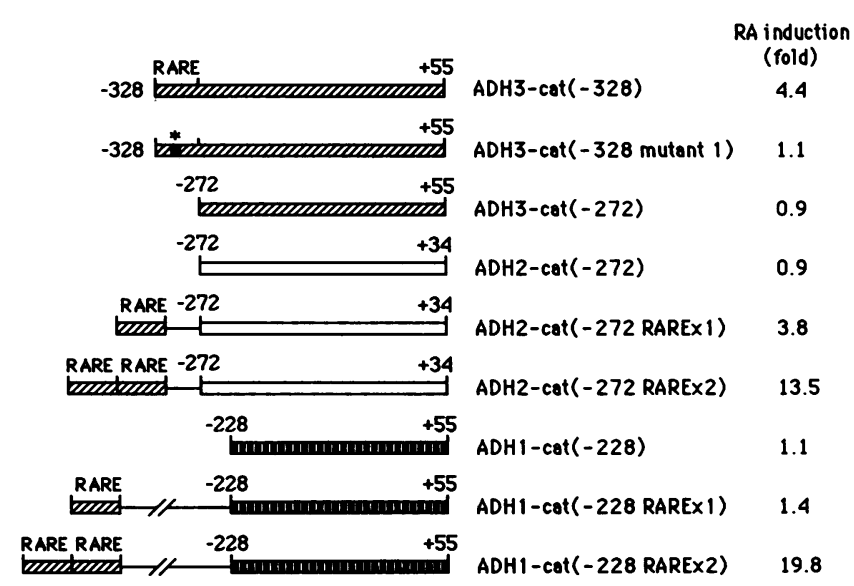

FIG. 4. Localization of the $A D H 3$ RARE by mutagenesis and heterologous promoter fusions. The various constructions shown were cotransfected with an RAR $\alpha$ expression vector in Hep3B cells with or without added retinoic acid, and the fold induction of CAT activity by retinoic acid for each construction is shown. The region indicated as a RARE in $A D H 3$ (from -328 to $-272 \mathrm{bp}$ ) was subjected to site-directed mutagenesis to give the construction ADH3-cat ( -328 mutant 1$)$, which has a block mutation at -289 to -285 (indicated by an asterisk). The $A D H 3$ RARE region from -328 to -272 bp (hatched bar) is shown fused in one or two copies to the $A D H 2$ promoter (open bar) as well as the $A D H 1$ promoter (striped bar). 


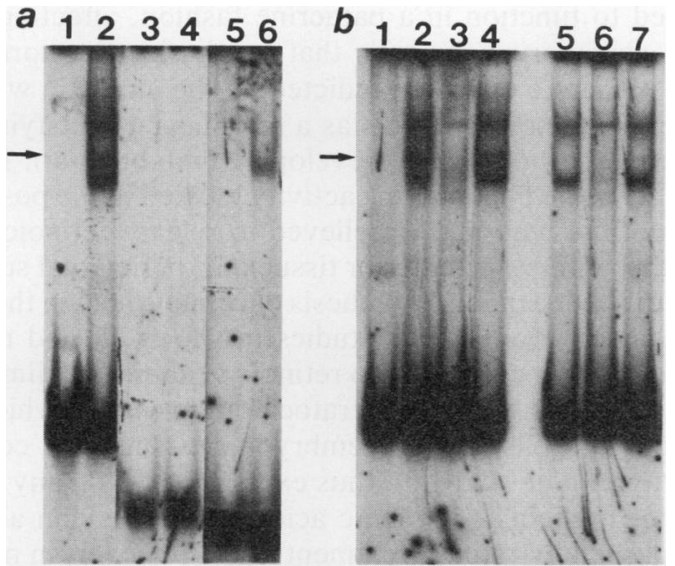

FIG. 5. Gel mobility shift analysis of the $A D H 3$ RARE. To examine potential interactions of the $A D H 3$ RARE with the RAR, ${ }^{32} \mathrm{P}$-labeled DNA probes were incubated in the presence or absence of a nuclear extract ( $4.5 \mu \mathrm{g}$ of protein), subjected to polyacrylamide gel electrophoresis, and analyzed by autoradiography. (a) Probes consisting of $A D H 3$ DNA from -328 to -272 bp (lanes 1 and 2), $A D H 3$ DNA from -328 to -290 bp (lanes 3 and 4), and RAR $\beta$ gene DNA from -59 to -33 bp (lanes 5 and 6) were incubated in the absence of nuclear extract (lanes 1,3, and 5) or in the presence of a nuclear extract derived from HeLa cells infected with a vaccinia virus-RAR $\beta$ expression vector (lanes 2,4 , and 6). (b) The $A D H 3$ probe containing -328 to -272 bp was treated with no nuclear extract (lane 1), with a vaccinia virus-RAR $\beta$-infected HeLa nuclear extract (lanes 2 to 4 ), or with an uninfected HeLa nuclear extract (lanes 5 to 7). A 100-fold molar excess of unlabeled RAR $\beta$ RARE oligonucleotide competitor was used (lanes 3 and 6), as well as a 100 -fold molar excess of unlabeled nonspecific competitor DNA (i.e., an oligonucleotide containing the HNF-3 binding site) (lanes 4 and 7). Arrows indicate the position of the -328 to -272-bp $A D H 3$ probe when specifically shifted by nuclear extract from vaccinia virus-RAR $\beta$-infected HeLa cells as opposed to uninfected HeLa cells. ized. The $A D H 3$ gene was demonstrated to possess a RARE in its 5 '-flanking region, whereas the closely related $A D H 1$ and $A D H 2$ genes were shown to lack such an element in the analogous regions of their 5'-flanking sequences. By using transient transfection and gel shift DNA-binding assays, the $A D H 3$ RARE was narrowed in location to the region between -328 to $-272 \mathrm{bp}$. This region conferred retinoic acid responsiveness upon the $A D H 1$ and $A D H 2$ promoters when fused to them. The sequence motif TGACC located between -289 to -285 of $A D H 3$ was shown to be of critical functional importance for the RARE, since its elimination by block substitution mutagenesis resulted in a lack of retinoic acid responsiveness of the $A D H 3$ promoter.

The $A D H 3$ RARE overlaps with a region containing a 29-bp directly repeated sequence that is highly diverged in the $A D H 1$ and $A D H 25^{\prime}$-flanking regions. The homologous regions of the $A D H 1, A D H 2$, and $A D H 3$ genes have been aligned to show the nucleotide sequence divergence (Fig. 6a). The TGACC motif which is present in both copies of the $A D H 3$ 29-bp direct repeat has been shown to play an important role in the RAREs of the growth hormone gene (42) and the laminin $\mathrm{B} 1$ gene (45). The $A D H I$ gene contains one TGACC motif in its single 29-bp element (Fig. 6a) but fails to respond to retinoic acid. This finding suggests that more than one TGACC or TGACC-like motif is necessary for retinoic acid responsiveness. $A D H 3$ also contains one copy of the related sequence TGAAC in the downstream 29-bp repeat (Fig. 6a). Two directly repeated copies of TGAAC are found in a RARE located in the human RAR $\beta$ gene (7) as well as the mouse RAR $\beta$ gene (39). A striking homology between $A D H 3$ and RAR $\beta$ RAREs is shown in Fig. $6 \mathrm{~b}$. In both genes there is a TGAAC motif followed downstream by a 6-bp linker, and then another TGAAC motif (RARß) or a TGACC motif $(A D H 3)$. Overall there is a 15-of-21-bp match in the regions encompassing the RAREs of these two genes, with the critical motifs spaced approxi-

a.

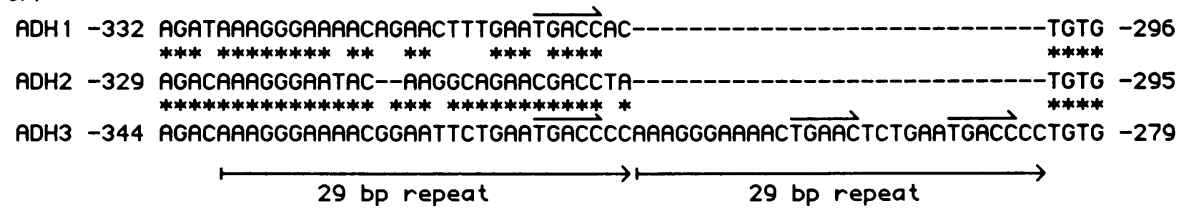

b. ADH3 -305 AAAAC $\overrightarrow{\mathrm{TGAACT}}$ TCTGAATGACCCCTGTGG -278

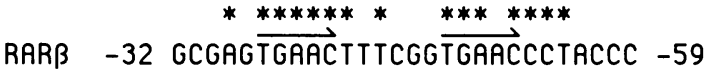
$15 / 21$ bp match

C. ADH3 -318 $\overrightarrow{\text { TGACCCCAAAGGGAAAACTGAACTCTGAATGACC }}-285$

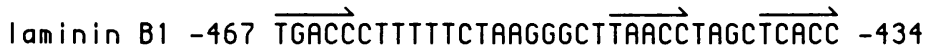

FIG. 6. Sequence homologies among $A D H 3$, RAR $\beta$, and laminin B1 RAREs. (a) Alignment of the nucleotide sequences of the 29-bp repeat regions of $A D H 1, A D H 2$, and $A D H 35^{\prime}$-flanking DNA (35). Asterisks indicate sequence identity among two or all three genes. The 29-bp monomer units, which form a direct repeat only in $A D H 3$, are indicated by long arrows. TGACC and TGAAC sequences, which are important RARE motifs in $A D H 3$ and other genes, are indicated by short arrows. (b) Alignment of the two downstream ADH3 RARE motifs with the RAR $\beta$ RARE, which has been shown to consist of two direct repeats of the TGAAC RARE motif $(7,39)$. In a 21-bp region encompassing both motifs, there is a 15-of-21-bp match in nucleotide sequence. (c) Alignment of all three ADH3 RARE motifs with three RARE motifs previously observed in the laminin B1 RARE (45). In both genes, the two motifs on the ends are spaced 29 bp apart, whereas the center motifs are offset by $2 \mathrm{bp}$. 


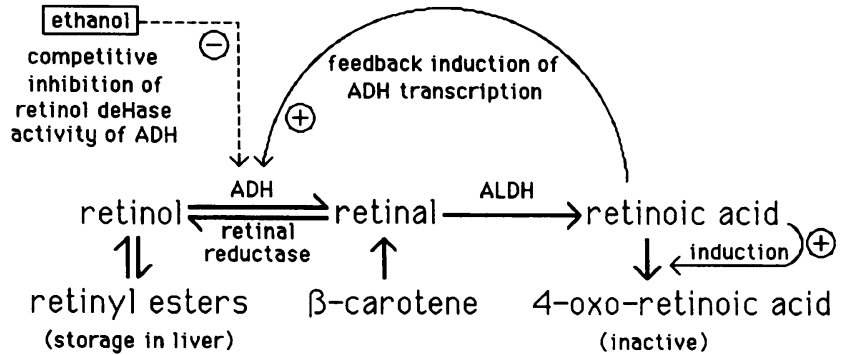

FIG. 7. Vitamin A homeostasis. Vitamin A is ingested as either $\beta$-carotene, retinol, or retinyl esters. $\beta$-Carotene is primarily broken down in the intestine to retinal, which is then converted to retinol by retinal reductase (13). Retinol is stored in the liver as retinyl esters, and the liver releases retinol to the serum in a controlled fashion to keep the concentration constant. Retinol in the liver, or retinol taken up by many other tissues, can be converted to retinoic acid via a two-step oxidation in which ADH (the rate-limiting step) produces retinal and aldehyde dehydrogenase (ALDH) produces retinoic acid $(22,26,48)$. Retinoic acid can be further oxidized to 4-oxo-retinoic acid as part of a degradation pathway for excretion, and the enzyme catalyzing this reaction is induced by retinoic acid (30). Studies reported here indicate that retinoic acid can also induce $A D H$ gene expression, suggesting a positive feedback mechanism for controlling retinoic acid synthesis. The effect of ethanol as a competitive inhibitor of $\mathrm{ADH}$ retinol oxidation $(21,26,29)$ is also indicated.

mately on the same side of the DNA helix 11 bp apart (Fig. $6 \mathrm{~b})$. In addition, $A D H 3$ and laminin B1 RAREs contain three TGACC or TGACC-like motifs spaced approximately equally in a 34-bp stretch (Fig. 6c). Our site-directed block mutation of the downstream TGACC sequence of $A D H 3$ located at -289 to -285 bp eliminated the retinoic acid response, as did a mutation of the homologous laminin B1 motif (45). Thus, RAREs controlling $A D H 3, \mathrm{RAR} \beta$, and laminin B1 gene transcription appear to share a great deal of sequence similarity, presumably providing a similar set of binding sites for retinoic acid receptors.

We have shown that the human $A D H 3$ gene contains a RARE that confers retinoic acid induction upon a linked promoter in a transfected hepatoma cell line. Since ADH catalyzes one of the steps in retinoic acid synthesis, we hypothesize that this may constitute a positive feedback loop regulating endogenous $A D H 3$ transcription and hence the level of retinoic acid in certain human cells. To achieve homeostasis of retinoic acid levels by using a positive feedback mechanism for stimulating synthesis, it might be necessary to couple this to a mechanism for degrading excess retinoic acid. Indeed, liver and other tissues possess an enzymatic activity, induced by retinoic acid, that oxidizes retinoic acid $\left(K_{m}=1 \mu \mathrm{M}\right)$ to the inactive 4-oxo derivative (30). Thus, retinoic acid feedback regulates its degradation as well as its synthesis. We present a scheme for achieving vitamin A homeostasis which combines previous knowledge of retinoid metabolism $(13,22,26,30,48)$ with our findings concerning positive feedback regulation of $A D H$ gene expression (Fig. 7).

According to this model, retinoic acid potentially functions as an intracrine hormone to self-regulate intracellular retinoic acid levels by controlling ADH (retinol dehydrogenase) gene expression. Intracrine regulation is defined as a mechanism whereby hormones are synthesized and act within a cell without the need for exit and reentry, as is the case for hormones that act by endocrine, paracrine, or autocrine mechanisms (27). Retinoic acid has also been proposed to function in a paracrine fashion, affecting cells closely neighboring the cells that synthesize the hormone. Such a paracrine effect is predicted by the model in which a gradient of retinoic acid acts as a morphogen specifying the anteroposterior axis of the developing limb bud (40). In this model, the zone of polarizing activity located in the posterior portion of the limb bud is believed to release retinoic acid, which then diffuses to anterior tissues. Experimental support for the existence of local synthesis of retinoic acid in the limb bud has been provided by studies in which labeled retinol was shown to be converted to retinoic acid in chick limb bud tissue (41). Also, mouse F9 teratocarcinoma cells, which are analogous to cells in early embryonic tissue, can convert retinol to retinoic acid (46), thus explaining why many of the well-known effects of retinoic acid on F9 cells can also be accomplished by retinol treatment (38). However, in neither of these studies was it determined whether class I ADH was the retinol dehydrogenase activity responsible for retinoic acid synthesis. The positive feedback mechanism that we propose for human $A D H 3$ gene expression potentially provides a very efficient method for localized synthesis of $\mathrm{ADH}$, and hence retinoic acid, and may be useful for establishing gradients of retinoic acid in certain tissues of the developing embryo. However, further studies are needed to determine whether $A D H 3$ is actually expressed in early human embryonic tissues and whether class I ADH or other enzymes (10) play the role of retinol dehydrogenase during retinoic acid synthesis in various tissues as well as various vertebrate species.

If class I ADH does play a role as a retinol dehydrogenase during human embryonic development, one can devise a molecular mechanism that helps explain the pathogenesis of fetal alcohol syndrome (10). Ethanol acts as a teratogen, causing abnormalities in nervous system and limb morphogenesis in those suffering from fetal alcohol syndrome (4, 37). Ethanol is a known substrate for all human class I ADH isozymes with $K_{m}$ values of approximately 1 to $2 \mathrm{mM}$ (26, 43), and it acts as a competitive inhibitor of human liver class I ADH retinol oxidation with a $K_{i}$ of $0.36 \mathrm{mM}$ (26). It is reasonable to propose that the teratogenic effects of ethanol on nervous system and limb morphogenesis may be mediated by a competitive substrate inhibition of the retinoloxidizing activity of fetal ADH, which would be expected to reduce the synthesis of retinoic acid known to play a critical role in morphogenesis of these tissues $(12,40,41)$. Such an inhibition may reduce the concentration of retinoic acid to a level that is insufficient to correctly specify spatial patterns in target tissues of the developing embryo.

\section{ACKNOWLEDGMENTS}

We thank H. Stunnenberg and M. Vivanco-Ruiz for providing a HeLa nuclear extract containing human RAR $\beta$ overexpressed in a vaccinia virus expression system as well as an oligonucleotide containing the RAR $\beta$ RARE; R. Evans for plasmids pRShRR, $\mathrm{p} \Delta \mathrm{MTV}-\mathrm{CAT}$, and $\mathrm{p} \Delta \mathrm{MTV}$-TRE-CAT; R. Costa for an oligonucleotide containing the HNF-3 binding site; and L. Winter, P. P. Harding, and C. Vanooij for technical assistance. We are grateful to J. Goliger and G. Vasios for pointing out the striking similarity between the RAREs of $A D H 3$ and laminin B1.

This study was supported by Public Health Service grant AA07261 from the National Institute on Alcohol Abuse and Alcoholism and by a BRSG award from the National Institutes of Health. G. D. is a recipient of Research Scientist Development Award K02 AA00119 from the National Institute on Alcohol Abuse and Alcoholism. 


\section{REFERENCES}

1. Arriza, J. L., C. Weinberger, G. Cerelli, T. M. Glaser, B. L. Handelin, D. E. Housman, and R. M. Evans. 1987. Cloning of human mineralocorticoid receptor complementary DNA: structural and functional kinship with the glucocorticoid receptor. Science 237:268-275.

2. Bilanchone, V., G. Duester, Y. Edwards, and M. Smith. 1986. Multiple mRNAs for human alcohol dehydrogenase (ADH): developmental and tissue-specific differences. Nucleic Acids Res. 14:3911-3926.

3. Brand, N., M. Petkovich, A. Krust, P. Chambon, H. de Thé, A. Marchio, P. Tiollais, and A. Dejean. 1988. Identification of a second human retinoic acid receptor. Nature (London) 332:850 853.

4. Burd, L., and J. T. Martsolf. 1989. Fetal alcohol syndrome: diagnosis and syndromal variability. Physiol. Behav. 46:39-43.

5. Costa, R. H., D. R. Grayson, and J. E. Darnell, Jr. 1989. Multiple hepatocyte-enriched nuclear factors function in the regulation of transthyretin and alpha-1-antitrypsin genes. Mol. Cell. Biol. 9:1415-1425.

6. de Thé, H., A. Marchio, P. Tiollais, and A. Dejean. 1987. A novel steroid thyroid hormone receptor-related gene inappropriately expressed in human hepatocellular carcinoma. Nature (London) 330:667-670.

7. de Thé, H., M. D. M. Vivanco-Ruiz, P. Tiollais, H. Stunnenberg, and A. Dejean. 1990. Identification of a retinoic acid responsive element in the retinoic acid receptor $\beta$ gene. Nature (London) 343:177-180.

8. Dignam, J. D., R. M. Lebovitz, and R. G. Roeder. 1983. Accurate transcription initiation by RNA polymerase II in a soluble extract from isolated mammalian nuclei. Nucleic Acids Res. 11:1475-1489.

9. Dollé, P., E. Ruberte, P. Kastner, M. Petkovich, C. M. Stoner, L. J. Gudas, and P. Chambon. 1989. Differential expression of genes encoding $\alpha, \beta$ and gamma retinoic acid receptors and CRABP in the developing limbs of the mouse. Nature (London) 343:702-705.

10. Duester, G. 1991. Human liver alcohol dehydrogenase gene expression: retinoic acid homeostasis and fetal alcohol syndrome. In R. R. Watson (ed.), Alcohol and drug abuse reviews: liver pathology and drugs of abuse. Humana Press, Clifton, N.J., in press.

11. Duester, G., M. Smith, V. Bilanchone, and G. W. Hatfield. 1986 Molecular analysis of the human class I alcohol dehydrogenase gene family and nucleotide sequence of the gene encoding the $\beta$ subunit. J. Biol. Chem. 261:2027-2033.

12. Durston, A. J., J. P. M. Timmermans, W. J. Hage, H. F. J. Hendriks, N. J. De Vries, M. Heideveld, and P. D. Nieuwkoop. 1989. Retinoic acid causes an anteroposterior transformation in the developing central nervous system. Nature (London) 340: 140-144.

13. Frolik, C. A. 1984. Metabolism of retinoids, p. 177-208. In M. B. Sporn, A. B. Roberts, and D. S. Goodman (ed.), The retinoids, vol. 2. Academic Press, Inc., Orlando, Fla.

14. Giguere, V., E. S. Ong, P. Segui, and R. M. Evans. 1987. Identification of a receptor for the morphogen retinoic acid. Nature (London) 330:624-629.

15. Gorman, C. M., L. F. Moffat, and B. H. Howard. 1982. Recombinant genomes which express chloramphenicol acetyltransferase in mammalian cells. Mol. Cell. Biol. 2:1044-1051.

16. Graupner, G., K. N. Wills, M. Tzukerman, X.-K. Zhang, and M. Pfahl. 1989. Dual regulatory role for thyroid-hormone receptors allows control of retinoic-acid receptor activity. Nature (London) 340:653-656.

17. Herbomel, P., B. Bourachot, and M. Yaniv. 1984. Two distinct enhancers with different cell specificities coexist in the regulatory region of polyoma. Cell 39:653-662.

18. Knowles, B. B., C. C. Howe, and D. P. Aden. 1980. Human hepatocellular carcinoma cell lines secrete the major plasma proteins and hepatitis B surface antigen. Science 209:497-499.

19. Koivusalo, M., M. Baumann, and L. Uotila. 1989. Evidence for the identity of glutathione-dependent formaldehyde dehydroge- nase and class II alcohol dehydrogenase. FEBS Lett. 257:105109.

20. Kunkel, T. A., J. D. Roberts, and R. A. Zakour. 1987. Rapid and efficient site-specific mutagenesis without phenotypic selection. Methods Enzymol. 154:367-382.

21. Leo, M. A., C. Kim, and C. S. Lieber. 1987. NAD+-dependent retinol dehydrogenase in liver microsomes. Arch. Biochem. Biophys. 259:241-249.

22. Leo, M. A., C. Kim, N. Lowe, and C. S. Lieber. 1989. Increased hepatic retinal dehydrogenase activity after phenobarbital and ethanol administration. Biochem. Pharmacol. 38:97-103.

23. Mangelsdorf, D. J., E. S. Ong, J. A. Dyck, and R. M. Evans. 1990. Nuclear receptor that identifies a novel retinoic acid response pathway. Nature (London) 345:224-229.

24. Mårdh, G., A. L. Dingley, D. S. Auld, and B. L. Vallee. 1986. Human class II $(\pi)$ alcohol dehydrogenase has a redox-specific function in norepinephrine metabolism. Proc. Natl. Acad. Sci. USA 83:8908-8912.

25. Maxam, A. M., and W. Gilbert. 1980. Sequencing end-labeled DNA with base-specific chemical cleavages. Methods Enzymol. 65:499-560.

26. Mezey, E., and P. R. Holt. 1971. The inhibitory effect of ethanol on retinol oxidation by human liver and cattle retina. Exp. Mol. Pathol. 15:148-156.

27. O'Malley, B. W. 1989. Did eukaryotic steroid receptors evolve from intracrine gene regulators? Endocrinology 125:1119-1120. (Editorial.)

28. Petkovich, M., N. J. Brand, A. Krust, and P. Chambon. 1987. A human retinoic acid receptor which belongs to the family of nuclear receptors. Nature (London) 330:444-450.

29. Posch, K. C., W. J. Enright, and J. L. Napoli. 1989. Retinoic acid synthesis by cytosol from the alcohol dehydrogenase negative deermouse. Arch. Biochem. Biophys. 274:171-178.

30. Roberts, A. B., M. D. Nichols, D. L. Newton, and M. B. Sporn. 1979. In vitro metabolism of retinoic acid in hamster intestine and liver. J. Biol. Chem. 254:6296-6302.

31. Roberts, A. B., and M. B. Sporn. 1984. Cellular biology and biochemistry of the retinoids, p. 209-286. In M. B. Sporn, A. B. Roberts, and D. S. Goodman (ed.), The retinoids, vol. 2. Academic Press, Inc., Orlando, Fla.

32. Sambrook, J., E. F. Fritsch, and T. Maniatis. 1989. Molecular cloning: a laboratory manual, 2nd ed. Cold Spring Harbor Laboratory, Cold Spring Harbor, N.Y.

33. Schüle, R., K. Umesono, D. J. Mangelsdorf, J. Bolado, J. W. Pike, and R. M. Evans. 1990. Jun-Fos and receptors for vitamins $A$ and $D$ recognize a common response element in the human osteocalcin gene. Cell 61:497-504.

33a.Shean, M. L., and G. Duester. Unpublished data.

34. Smith, M., D. A. Hopkinson, and H. Harris. 1971. Developmental changes and polymorphism in human alcohol dehydrogenase. Ann. Hum. Genet. 34:251-271.

35. Stewart, M. J., M. S. McBride, L. A. Winter, and G. Duester. 1990. Promoters for the human alcohol dehydrogenase genes $A D H 1, A D H 2$, and $A D H 3$ : interaction of CCAAT/enhancer binding protein with elements flanking the $A D H 2$ TATA box. Gene 90:271-279.

36. Stewart, M. J., M. L. Shean, and G. Duester. 1990. transactivation of human alcohol dehydrogenase gene expression in hepatoma cells by C/EBP molecules bound in a novel arrangement just $5^{\prime}$ and $3^{\prime}$ to the TATA box. Mol. Cell. Biol. 10:50075010.

37. Streissguth, A. P., S. Landesman-Dwyer, J. C. Martin, and D. W. Smith. 1980. Teratogenic effects of alcohol in humans and laboratory animals. Science 209:353-361.

38. Strickland, S., and V. Mahdavi. 1978. The induction of differentiation in teratocarcinoma stem cells by retinoic acid. Cell 15:393-403.

39. Sucov, H. M., K. K. Murakami, and R. M. Evans. 1990. Characterization of an autoregulated response element in the mouse retinoic acid receptor type $\beta$ gene. Proc. Natl. Acad. Sci. USA 87:5392-5396.

40. Thaller, C., and G. Eichele. 1987. Identification and spatial distribution of retinoids in the developing chick limb bud. 
Nature (London) 327:625-628.

41. Thaller, C., and G. Eichele. 1988. Characterization of retinoid metabolism in the developing chick limb bud. Development 103:473-483.

42. Umesono, K., V. Giguere, C. K. Glass, M. G. Rosenfeld, and R. M. Evans. 1988. Retinoic acid and thyroid hormone induce gene expression through a common responsive element. Nature (London) 336:262-265.

43. Vallee, B. L., and T. J. Bazzone. 1983. Isozymes of human liver alcohol dehydrogenase, p. 219-244. In M. C. Rattazzi, J. G. Scandalios, and G. S. Whitt (ed.), Isozymes, vol 8. Cellular localization, metabolism, and physiology. Alan R. Liss, Inc., New York.

44. Van Thiel, D. H., J. Gavaler, and R. Lester. 1974. Ethanol inhibition of vitamin A metabolism in the testes: possible mechanism for sterility in alcoholics. Science 186:941-942.

45. Vasios, G. W., J. D. Gold, M. Petkovich, P. Chambon, and L. J. Gudas. 1989. A retinoic acid-responsive element is present in the 5 ' flanking region of the laminin B1 gene. Proc. Natl. Acad. Sci. USA 86:9099-9103.

46. Williams, J. B., and J. L. Napoli. 1985. Metabolism of retinoic acid and retinol during differentiation of F9 embryonal carcinoma cells. Proc. Natl. Acad. Sci. USA 82:4658-4662.

47. Winter, L. A., M. J. Stewart, M. L. Shean, Y. Dong, L. Poellinger, S. Okret, J.-Å. Gustafsson, and G. Duester. 1990. A hormone response element upstream of the human alcohol dehydrogenase gene $A D H 2$ consists of three tandem glucocorticoid receptor binding sites. Gene 91:233-240.

48. Zachman, R. D., and J. A. Olson. 1961. A comparison of retinene reductase and alcohol dehydrogenase of rat liver. $\mathbf{J}$. Biol. Chem. 236:2309-2313.

49. Zelent, A., A. Krust, M. Petkovich, P. Kastner, and P. Chambon. 1989. Cloning of murine $\alpha$ and $\beta$ retinoic acid receptors and a novel receptor gamma predominantly expressed in skin. Nature (London) 339:714-721. 